\title{
Relationship between Sex-role Identity and Functional Cerebral Lateralization
}

\author{
Tomoaki Kozaki and Akira Yasukouchi \\ Department of Physiological Anthropology, Faculty of Design, Kyushu University
}

\begin{abstract}
The present study examined the relationship between sex-role identity (SRI) and functional cerebral lateralization (FCL) in right-handed males. Two tasks (figure task and location task) were used to assess FCL. The figure task required the identification of shape stimuli, while the location task involved identification of the position of stimuli. SRI was assessed by the Bem Sex Role Inventory (BSRI). Males with higher masculine scores in the BSRI indicated greater differences in the reaction time between right and left visual-fields in the location task. This finding suggests that males with higher masculinity in SRI might have greater FCL. J Physiol Anthropol Appl Human Sci 24(3): 221-225, 2005 http://www.jstage.jst.go.jp/browse/jpa

[DOI: 10.2114/jpa24.221]
\end{abstract}

Keywords: sex-role identity, functional cerebral lateralization, visual task, reaction time, right-handed males

\section{Introduction}

Males may have greater functional cerebral lateralization (FCL) compared with females (Kimura, 1992; Kikuchi, 1998) in humans. Although sexual differences in FCL have been shown in functional magnetic resonance imaging (fMRI) studies (Levin et al., 1998; Baxter et al., 2003), greater FCL in males has not been reported in fMRI responses (Gur et al., 2000). A review of visual half-field experiments has revealed no differences in performance between right and left visualfields in males (Hiscock et al., 1995). These studies are inconsistent with the finding that greater FCL occurs in males.

Sex-role identity (SRI) may be related to FCL. Sex steroidal hormones have been identified as a major factor in sexual differentiation of brain function (Toran-Allerand, 1984; Kimura, 1992; Kikuchi, 1998). On reviewing the relationships between sex steroidal hormones and FCL, Wisniewski (1998) concluded that testosterone is the most likely candidate to influence FCL. It has been thought that testosterone (androgen) plays a role in mascularizing SRI (Dörner, 1985; Wilson, 1999, 2001). Baucom et al. (1985) have reported that females with high masculinity levels display higher testosterone levels than those with low masculinity levels. Moreover, a positive relationship between masculine scores and spatial performance has been observed (Hamilton, 1995). In comparison with homosexual males, heterosexual males have shown better spatial performance (Sanders and Wright, 1997), demonstrating that a significant difference exists between the left and right visual-field scores in heterosexual males, although the visual difference was not significant for homosexual males. Those findings imply that males with a higher masculine level have greater FCL.

The present study was designed to investigate the relationship between SRI and FCL in right-handed males. We evaluated FCL by visual tasks, where a stimulus was delivered to the peripheral visual-field, because semi-field visual information is relayed en masse to the contralateral visual cortex via the chiasm (Wolter, 1965). Since it has been suggested that testosterone accelerates information processing of the right cerebral hemisphere (Tan et al., 1993), two visual tasks espousing identification of the shape (figure task) and position (location task) of a target-stimulus were performed. The location task would strongly involve information processing of the right cerebral hemisphere, as this cerebral hemisphere dominates the functional aspects of visuo-spatial attention and visuo-spatial information processing (Heilman and Van Den Abell, 1980; Corbetta et al., 1993; Nobre et al., 1997; Butler et al., 2004).

\section{Methods}

\section{Subjects}

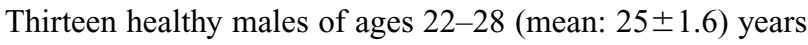
participated in the study. All subjects were assessed as righthanded by the Chapman Handedness Inventory (Chapman and Chapman, 1987).

\section{Apparatus}

A personal computer (Pro-650, EPSON DIRECT Co. Ltd., Japan) with an 18-inch LCD display (Flex Scan L676, EIZO NANAO Co. Ltd., Japan) and a timer board (PC-6301, Interface Co. Ltd., Japan) was installed with a mouse. Subjects used a chin-rest to maintain a consistent distance of $56 \mathrm{~cm}$ from the eye to the display panel at variable visual angles. 
a: Figure task

Prediction

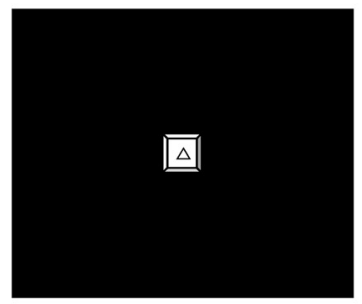

$[O]$ or $[\Delta]$ or $[\square]$

b: Location task

Prediction and Landmark

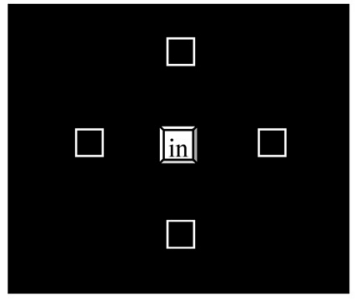

[In] or [Out]

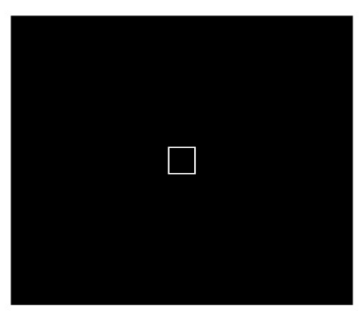

$2000 \sim 4000 \mathrm{~ms}$
Target

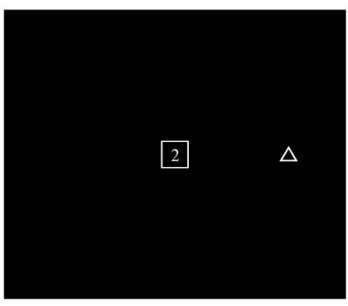

$100 \mathrm{~ms}$
Digit Report Form

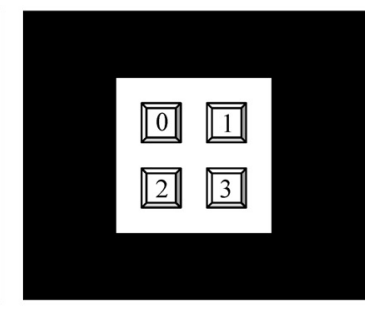$$
\text { 政 }
$$

Target

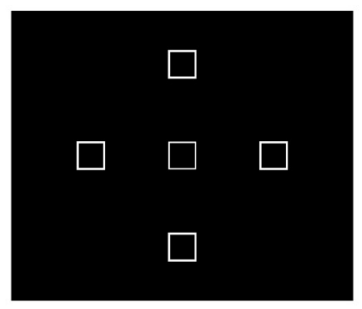

2000 4000ms

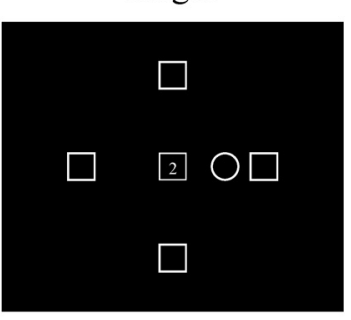

$100 \mathrm{~ms}$

Fig. 1 These panels symbolize the sequence of display for the two visual tasks.

\section{Visual tasks}

For the figure task (Fig. 1a), a prediction stimulus (circle, triangle or quadrangle; visual angle: $0.5^{\circ}$ from the horizontal plane) was presented in the center of the display. Subjects were required to click the stimulus with the mouse, whereupon it disappeared. After the stimulus had disappeared, a fixation box (visual angle: $0.5^{\circ}$; see above) appeared in the center of the display panel for $2000-4000 \mathrm{~ms}$ followed by display of a target-stimulus (circle, triangle or quadrangle; visual angle: $0.5^{\circ}$; see above) with a digit $\left(0,1,2\right.$ or 3 ; visual angle: $\left.0.3^{\circ}\right)$ for $100 \mathrm{~ms}$. The target-stimulus was located at $3^{\circ}$ or $6^{\circ}$ from the digit in the fixation box. The subject was instructed to press a button on the mouse as quickly as possible, and the reaction time (RT) was clocked when the target-stimulus was identical with the prediction stimulus. Finally, a report form with digits appeared, and subjects keyed in the digit detected by clicking the corresponding digit on the display.

The location task was conducted in a manner similar to the figure detection task. In the location task (Fig. 1b), landmark stimuli (four quadrangles; visual angle $0.5^{\circ}$; see above) were presented at $3^{\circ}$ or $6^{\circ}$ from a prediction stimulus ("in" or "out"; 10 -point Gothic font). A target-stimulus (circle; visual angle $0.5^{\circ}$; see above) appeared at a location, either central (in) or peripheral (out) from the landmark stimulus. Subjects were then required to detect the location of the target-stimulus.

In both tasks, it was confirmed that the subjects could detect the target-stimuli of each location before the experiment.

\section{Bem Sex Role Inventory (BSRI)}

The BSRI (Bem, 1974) was used to assess masculine personality characteristics. Of the 60 items in the BSRI, 20 items each within the inventory were concerned with masculine and feminine characteristics, respectively. Subjects were required to rate themselves using a 7-point scale on each item. The scale ranged from 1 (never true) to 7 (always true).

\section{Design and procedure}

The figure and location tasks consisted of 384 and 128 trials, respectively. The former was separated into 6-block trials and while the latter into 2-block trials, with each block consisting of 64 trials. In each task, $75 \%$ of trials with the target-stimulus presented the same shape as the predicted stimuli (valid trials), and the remaining $25 \%$ of trials were presented in different shapes (invalid trials). Trials of validity and stimulus were randomized within each block. When subjects reported a digit different from the presented digit, the trials were then repeated after the last trial of the block, because the subjects were considered not to have focused on the fixation box. If the error rate of the response in target discrimination exceeded $10 \%$, the block was repeated.

Three blocks for the figure task and one block for the location task were performed daily. Subjects performed the four blocks of visual tasks in an order that virtually counterbalanced cross-effects on the subjects, and all blocks were completed in 2-3 days. The BSRI was measured after the completion of all visual tasks. 


\section{Data analysis}

RTs for the upper and lower visual-fields were not analyzed, and those in error trials and invalid trials were omitted from the data analysis. Furthermore, RTs which were below the average by $-2 \times$ S.D. or above the average by $+2 \times$ S.D. of total RTs were excluded. RTs for the right and left visual-fields were averaged separately.

The masculinity score was calculated by summating the scores within the masculine subscale on the BSRI.

\section{Results}

Mean masculinity scores in this study $(86.54 \pm 11.88$; mean \pm S.D.) were slightly lower than the previous values (91.21 15.99; Azuma, 1991) established with the BSRI in 307 young Japanese males. The relationship between masculinity scores on the BSRI and $\Delta \mathrm{RT}(\mathrm{RT}$ difference $=$ left visual-field RT-right visual-field RT) could be best described by a quadratic equation in the location task (Fig. 2). In the figure task, there was no significant relationship between

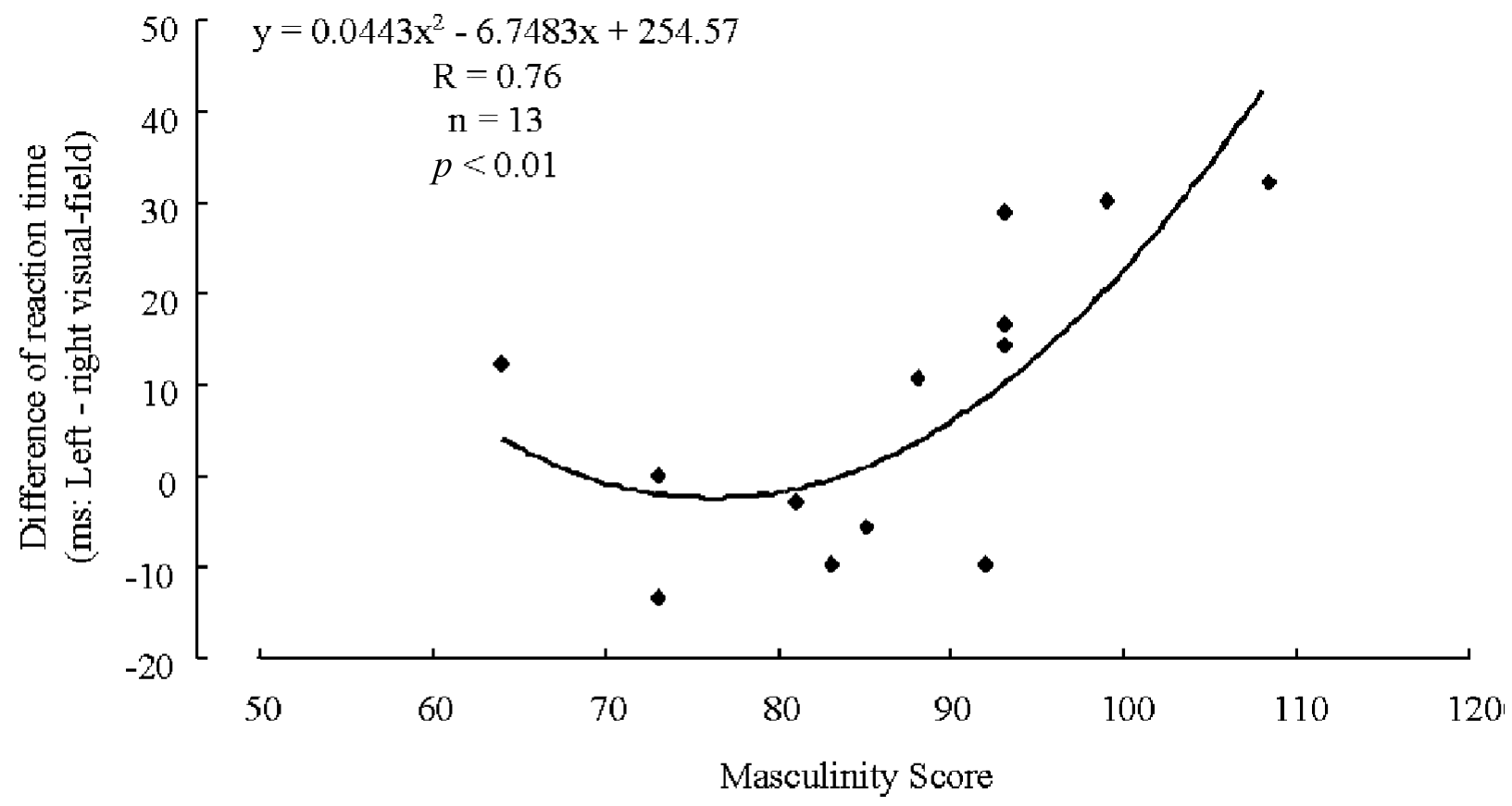

Fig. 2 Relationship between masculine scores and the RT differences in the location task

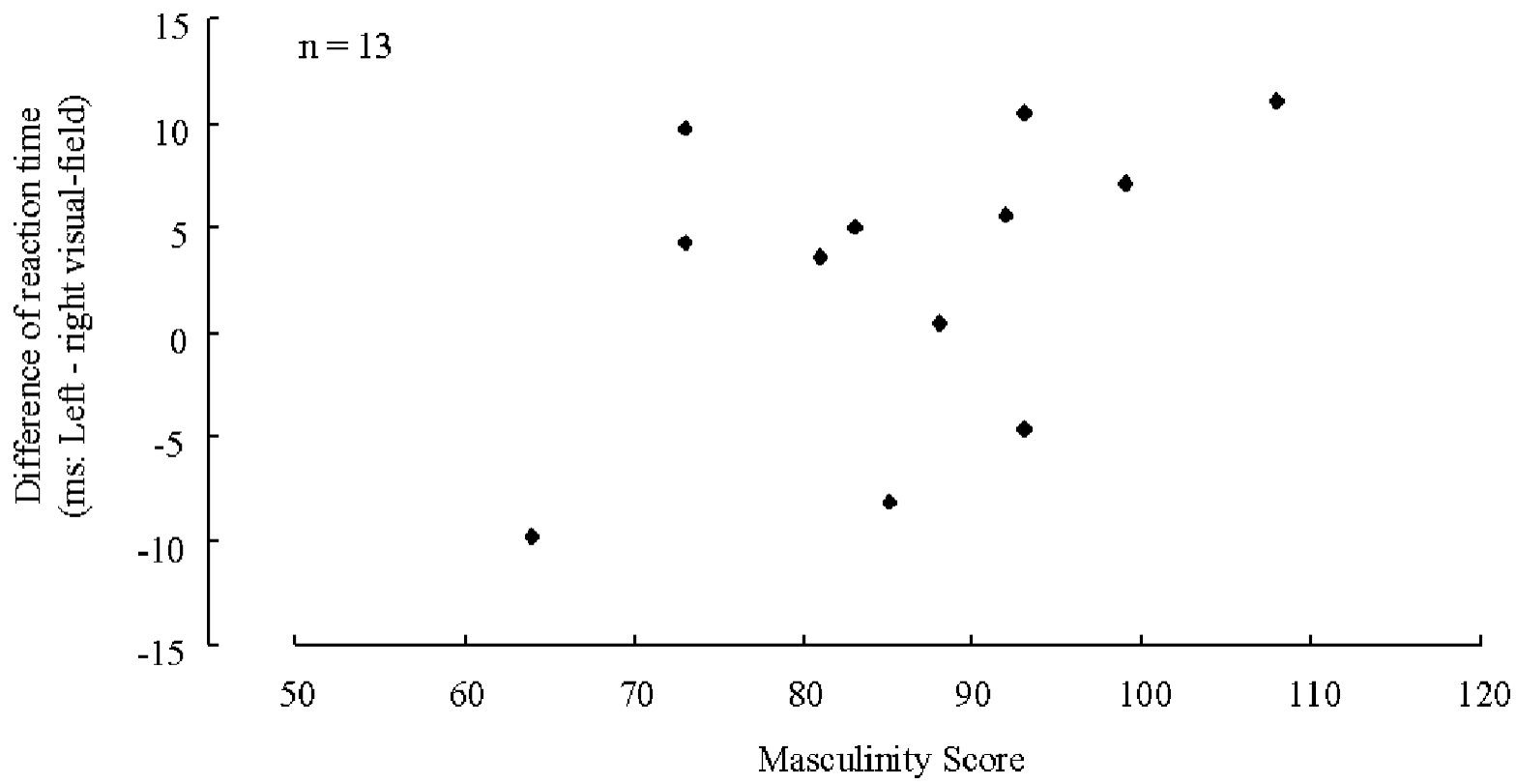

Fig. 3 Relationship between masculine scores and the RT differences in the figure task. 
masculinity scores on the BSRI and $\Delta$ RT (Fig. 3).

\section{Discussion}

While our results demonstrated a significant relationship between masculinity scores and $\Delta \mathrm{RT}$ in location tasks, significant relationships between the two parameters were not established in figure tasks. The location task is considered to involve visuo-spatial attention and visuo-spatial informationprocessing, functions which are predominant in the right cerebral hemisphere (Heilman and Van Den Abell, 1980; Corbetta et al., 1993; Nobre et al., 1997; Butler et al., 2004). As such, the present findings suggest that males with a high masculinity level have greater FCL for visuo-spatial attention and/or visuo-spatial information-processing.

However, $\triangle \mathrm{RT}$ in location tasks would not have been induced by FCL in visuo-spatial information-processing. Testosterone (androgens) has been thought of as the main factor contributing to masculinization of SRI (Baucom et al., 1985; Berenbaum et al., 2000) and accelerating informationprocessing in the right hemisphere (Tan et al., 1993). If testosterone were to accelerate information-processing in the right hemisphere, the RT to stimuli in the left would be faster than the right visual-field. Conversely, our results indicated shorter RTs to stimuli in the right than the left visual-field in location tasks, suggesting that testosterone could not have accelerated visuo-spatial information-processing in the right hemisphere.

The predominant contribution from the right hemisphere to visuo-spatial attention might induce a shorter RT to the right visual-field in males with higher masculinity levels. It has been demonstrated that visuo-spatial attention directed to the right visual-field activates both cerebral hemispheres (Corbetta et al., 1993; Nobre et al., 1997), albeit that the same attention directed to the left visualfield only activates the right cerebral hemisphere (Corbetta et al., 1993; Heinze et al., 1994; Mangun et al., 1997). Hikosaka et al. (1993a, b) have reported a facilitatory effect of visuo-spatial attention on visual information-processing. Furthermore, Rhodes and Robertson (2002) have found a significantly shorter RT to the right visualfield in valid precue trials, whereas there was no significant $\Delta \mathrm{RT}$ for invalidly precued targets. They concluded that the allocation of visuo-spatial attention affects processing asymmetries for a visual target. Those studies suggest that visual information-processing of the right visual-field may be facilitated by visuo-spatial attention in both hemispheres, yielding a shorter RT to the right visual-field. Males with higher masculinity levels have been reported to display superior spatial ability compared with those with lower masculinity levels (Hamilton, 1995). Moreover, Voyer and Bryden (1990) have observed a significantly shorter RT to the right visual-field in subjects with higher spatial ability. Those findings might support the idea that males with higher masculinity levels have predominance of the right hemisphere for visuo-spatial attention.

This study provides an interesting finding in the relationship between SRI and FCL. However, the present findings might not be replicated in left-handed subjects or in other cultures. A review of cross-cultural perspectives inferred that handedness is probably associated with laterality of brain function (Dawson, 1977); viz., current traditional agropopulations such as the Hong Kong Chinese have a significantly lower incidence of left-handers compared with Inuit hunters. The study further advocates that the agropopulation places greater emphasis on conformity in the socialization of females than doInuit hunters. Therefore, sociocultural factors might have molded the relationship between SRI and FCL. Additionally, according to Moffat and Hampson (1996), right-handed subjects of both sexes indicate a significantly larger right ear advantage than left-handed subjects in the dichotic listening task. Further quantitative studies of sex steroidal hormones and comparative elucidations of handedness and cultural factors correlated with the SRI-FCL relationships are warranted.

Acknowledgments This research was supported in part by a Grant-in-Aid for Scientific Research (S: No. 15107006) from Japan Society for the Promotion of Science (JSPS) and a Grant-in-Aid for the 21st Century COE Program from the Ministry of Education, Culture, Sports, Science and Technology (MEXT).

\section{References}

Azuma K (1991) Psychological Androgyny II. Academic Studies. Education 40: 61-71 [In Japanese]

Baucom DH, Besch PK, Callahan S (1985) Relation between testosterone concentration, sex role identity, and personality among females. J Pers Soc Psychol 48: 1218-1226

Baxter LC, Saykin AJ, Flashman LA, Johnson SC, Guerin SJ, Babcock DR, Wishart HA (2003) Sex differences in semantic language processing: a functional MRI study. Brain Lang 84: 264-272

Bem SL (1974) The measurement of psychological androgyny. J Consult Clin Psychol 42: 155-162

Berenbaum SA, Duck SC, Bryk K (2000) Behavioral effects of prenatal versus postnatal androgen excess in children with 21-hydroxylase-deficient congenital adrenal hyperplasia. J Clin Endocrinol Metab 85: 727-733

Butler AJ, Fink GR, Dohle C, Wunderlich G, Tellmann L, Seitz RJ, Zilles K, Freund HJ (2004) Neural mechanisms underlying reaching for remembered targets cued kinesthetically or visually in left or right hemispace. Hum Brain Mapp 21: 165-177

Chapman LJ, Chapman JP (1987) The measurement of handedness. Brain Cogn 6: 175-83

Corbetta M, Miezin FM, Shulman GL, Petersen SE (1993) A PET study of visuo spatial attention. J Neurosci 13: 12021226

Dawson JL (1977) An anthropological perspective on the 
evolution and lateralization of the brain. Ann NY Acad Sci 299: 424-447

Dörner G (1985) Sex-specific gonadotrophin secretion, sexual orientation and gender role behaviour. Exp Clin Endocrinol 86: $1-6$

Gur RC, Alsop D, Glahn D, Petty R, Swanson CL, Maldjian JA, Turetsky BI, Detre JA, Gee J, Gur RE (2000) An fMRI study of sex differences in regional activation to a verbal and a spatial task. Brain Lang 74: 157-170

Hamilton CJ (1995) Beyond sex differences in visuo-spatial processing: the impact of gender trait possession. $\mathrm{Br} \mathrm{J}$ Psychol 86 (Pt 1): 1-20

Heilman KM, Van Den Abell T (1980) Right hemisphere dominance for attention: the mechanism underlying hemispheric asymmetries of inattention (neglect). Neurology 30: 327-330

Heinze HJ, Mangun GR, Burchert W, Hinrichs H, Scholz M, Münte TF, Gös A, Scherg M, Johannes S, Hundeshagen H, Gazzaniga MS, Hillyard SA (1994) Combined spatial and temporal imaging of brain activity during visual selective attention in humans. Nature 372: 543-546

Hikosaka O, Miyauchi S, Shimojo S (1993a) Focal visual attention produces illusory temporal order and motion sensation. Vision Res 33: 1219-1240

Hikosaka O, Miyauchi S, Shimojo S (1993b) Voluntary and stimulus-induced attention detected as motion sensation. Perception 22: 517-526

Hiscock M, Israelian M, Inch R, Jacek C, Hiscock-Kalil C (1995) Is there a sex difference in human laterality? II. An exhaustive survey of visual laterality studies from six neuropsychology journals. J Clin Exp Neuropsychol 17: 590-610

Kikuchi Y (1998) Male brain and female brain. Appl Human Sci 17: 161-163

Kimura D (1992) Sex differences in the brain. Sci Am 267: 118-25

Levin JM, Ross MH, Mendelson JH, Mello NK, Cohen BM, Renshaw PF (1998) Sex differences in blood-oxygenationlevel-dependent functional MRI with primary visual stimulation. Am J Psychiatry 155: 434-436

Mangun GR, Hopfinger JB, Kussmaul CL, Fletcher EM, Heinze HJ (1997) Covariations in ERP and PET measures of spatial selective attention in human extrastriate visual cortex. Hum Brain Map 5: 273-279
Moffat SD, Hampson E (1996) Salivary testosterone levels in left- and right-handed adults. Neuropsychologia 34: 225233

Nobre AC, Sebestyen GN, Gitelman DR, Mesulam MM, Frackowiak RS, Frith CD (1997) Functional localization of the system for visuo spatial attention using positron emission tomography. Brain 120: 515-533

Rhodes DL, Robertson LC (2002) Visual field asymmetries and allocation of attention in visual scenes. Brain Cogn 50: 95-115

Sanders G, Wright M (1997) Sexual orientation differences in cerebral asymmetry and in the performance of sexually dimorphic cognitive and motor tasks. Arch Sex Behav 26: 463-480

Tan Ü, Akgun A, Komsuoglu S, Telatar M (1993) Inverse relationship between nonverbal intelligence and the parameters of pattern reversal visual evoked potentials in left-handed male subjects: importance of right brain and testosterone. Int J Neurosci 71: 189-200

Toran-Allerand CD (1984) Gonadal hormones and brain development: implications for the genesis of sexual differentiation. Ann NY Acad Sci 435: 101-111

Voyer D, Bryden MP (1990) Gender, level of spatial ability, and lateralization of mental rotation. Brain Cogn 13: 18-29

Wilson JD (1999) The role of androgens in male gender role behavior. Endocr Rev 20: 726-737

Wilson JD (2001) Androgens, androgen receptors, and male gender role behavior. Horm Behav 40: 358-366

Wisniewski AB (1998) Sexually-dimorphic patterns of cortical asymmetry, and the role for sex steroid hormones in determining cortical patterns of lateralization. Psychoneuroendocrinol 23: 519-547

Wolter JR (1965) The centrifugal nerves in the human optic tract, chiasm, optic nerve, andretina. Trans Am Ophthalmol Soc 63: 678-707

Received: February 12, 2004

Accepted: March 2, 2005

Correspondence to: Tomoaki Kozaki, Department of Physiological Anthropology, Faculty of Design, Kyushu University 4-9-1 Shiobaru, Minami-ku, Fukuoka 815-8540, Japan

Phone: +81-92-553-4530

Fax: +81-92-553-4530

e-mail: kozaki@design.kyushu-u.ac.jp 